\title{
Taguchi optimization and ultrasonic measurement of residual stresses in the friction stir welding
}

\author{
Yashar Javadi ${ }^{1 *}$ \\ ${ }^{1}$ Department of Mechanical Engineering, Semnan Branch, Islamic Azad University, Semnan, Iran. \\ ( ${ }^{*}$ Corresponding author's e-mail: yashar_javadi@aut.ac.ir; Tel: +98 9124402303, Fax: +98 231335 4030.) \\ Seyedali Sadeghi ${ }^{2}$ \\ ${ }^{2}$ Department of Mechanical Engineering, Amirkabir University of Technology, 424 Hafez Ave.,15914, Tehran, Iran. \\ sadeghi1368@aut.ac.ir \\ Mehdi Ahmadi Najafabadi ${ }^{3}$ \\ ${ }^{3}$ Department of Mechanical Engineering, Amirkabir University of Technology, 424 Hafez Ave.,15914, Tehran, Iran. \\ ahmadin@aut.ac.ir
}

\begin{abstract}
The main goal of this study is optimization of residual stresses produced by friction stir welding (FSW) of 5086 aluminum plates. Taguchi method is employed as statistical design of experiment (DOE) to optimize welding parameters including feed rate, rotational speed, pin diameter and shoulder diameter. The optimization process depends on effect of the welding parameters on longitudinal residual stress, which is measured by employing ultrasonic technique. The ultrasonic measurement method is based on acoustoelasticity law, which describes the relation between acoustic waves and internal stresses of the material. In this study, the ultrasonic stress measurement is fulfilled by using longitudinal critically refracted $\left(L_{C R}\right)$ waves which are longitudinal ultrasonic waves propagated parallel to the surface within an effective depth. The ultrasonic stress measurement results are also verified by employing the hole-drilling standard technique. By using statistical analysis of variance (ANOVA), it has been concluded that the most significant effect on the longitudinal residual stress peak is related to the feed rate while the pin and shoulder diameter have no dominant effect. The rotational speed variation leads to changing the welding heat input which affect on the residual stress considerably.
\end{abstract}


Keywords: Friction Stir Welding; Taguchi method; Ultrasonic Stress Measurement; Welding Residual Stress; Acoustoelasticity.

\section{Introduction}

\subsection{Welding residual stress}

Residual stresses are known as remaining stresses inside the material after manufacturing process, in the absence of any external loads or thermal gradients. The engineering properties of industrial equipment particularly fatigue life, dimensional stability, corrosion resistance, and brittle fracture can be considerably influenced by residual stresses. Welding processes, as essential production processes in the industry, produces residual stresses at a significant level. Welding residual stresses are the results of non-uniform thermal expansions and solidifications caused by the welding processes.

\subsection{Residual stress measurement}

Residual stresses measurement is a critical stage in the structures design and also in the reliability estimation of mechanical equipment under real service conditions. There are various methods available for stress measurement including three main categories: destructive, semidestructive and non-destructive methods [1]. The destructive and semi destructive techniques, also called as mechanical method, rely on measuring the deformations produced by releasing residual stresses upon material removal from the specimen. Sectioning and contour method are principal destructive techniques which completely destruct the specimen to evaluate the residual stresses while hole-drilling, ring-core and deep-hole-drilling are semi-destructive techniques leave small holes on the material surface. Among them, the hole-drilling method is one of the most popular methods providing the stress measurement with good accuracy and reliability. Since the hole-drilling method is standardized by ASTM: E837, it is used by the majority of studies as a verification of other stress measurement methods [2-6]. Non-destructive methods including X-ray diffraction, neutron diffraction, ultrasonic and magnetic methods usually measure some parameter that is related to the stress. The non-destructive evaluation of stress becomes increasingly important since many structural components, e.g. bridges, aircraft 
structures or offshore platforms, need to be inspected periodically to avoid major damage or even failure.

\subsection{Ultrasonic stress measurement}

Ultrasonic stress measurement is founded on the linear relation between velocity of the ultrasonic wave and the material stress. This correlation, within the elastic limit, is known as the acoustoelastic effect, which says that flight time of the ultrasonic wave changes with the stress. In 1967, Crecraft [7] showed that the acoustoelastic law could be employed for stress measurement of engineering materials. The longitudinal critically refracted $\left(L_{C R}\right)$ wave is a longitudinal ultrasonic wave propagated parallel to the surface. It has been proved by Egle and Bray [8] that the $L_{C R}$ wave sensitivity to the stress is highest among the other types of ultrasonic waves. Tang and Bray [9] utilized the $L_{C R}$ waves to measure the stresses and also studied effect of plastic deformation on the wave velocity variations. The $L_{C R}$ waves were employed to evaluate bending stress in steel plates and bars by Bray and Tang [10]. They used two different testing frequencies (2.25 MHz and $5 \mathrm{MHz}$ ) and compared the results. They tried to confirm a unique capability of the ultrasonic method, which is penetrating in different depth (by changing the frequency of transducers) and measuring bulk stress in different depths. It means that the ultrasonic stress measurement could be used to measure through-thickness stresses while the aforementioned capbility was also confirmed by Javadi et al. [11] and Sadeghi et al [12] in the stainless steel and aluminum plates, respectively. By employing the $L_{C R}$ waves produced by four different frequencies (1 MHz, $2 \mathrm{MHz}, 4 \mathrm{MHz}$ and $5 \mathrm{MHz}$ ) of ultrasonic transducers, Sadeghi et al [12] determined distribution of longitudinal residual stress through the thickness of aluminum plates joined by friction stir welding. Javadi et al [13] employed the $L_{C R}$ method to measure through-thickness distribution of hoop and axial residual stresses in stainless steel pipes. However, they recommended using another stress measurement method (like hole-drilling method) to be used as verification of the $L_{C R}$ measurement method.

The $L_{C R}$ measurement was developed in immersion mode by Belahcene and Lu [14] to measure welding residual stresses of S355 steel plate. They used hole-drilling method to verify the ultrasonic stress measurements. Palanichamy et al [15] measured the residual stresses in austenitic stainless steel weld joints using ultrasonic technique. Javadi et al [16] presented a comparison between using contact and immersion transducers in ultrasonic stress measurement 
of dissimilar plates and pipes. In another work, they also compared contact and immersion mode in ultrasonic stress measurement of stainless steels plates [17]. Comparing the results obtained from aforementioned works ([16] and [17]) shows that there is no considerable difference between using the contact and immersion technique, hence the contact transducers are employed in this study.

Recently, employing the $L_{C R}$ waves is more popular in the ultrasonic stress measurement and the majority of studies consider the longitudinal waves more than the ultrasonic shear waves [1824]. Hence, the $L_{C R}$ waves are used in this study to measure the residual stresses of the aluminum plates joined by friction stir welding.

\subsection{Friction stir welding (FSW)}

The welding residual stresses may be produced after all of the welding processes including both fusion and solid state welding processes. Solid-state welding processes do not involve the melting of the structures being joined; instead, the weld is created by producing coalescence at temperatures below the melting point of the base metals, without addition of the filler metal. Friction stir welding (FSW) is a solid state welding process patented by TWI [25], in which a

rotating tool (with a pre-determined tilt angle) is embedded into the adjoining edges of the plates to be welded, and then moved all along the welded joint. As the tool moves, the material is forced to flow around the tool in a complex flow pattern, which has been described in several literatures [26-28]. The result would be producing frictional heating along with plastic deformation in the welding zone while no material melting is created.

Bussu and Irving [29] showed that the fatigue life is improved by presence of the residual stresses; however they considered distortions caused by the residual stresses that restrict application of the FSW process in manufacturing production. Peel et al. [30] employed the X-ray diffraction method to evaluate distribution of residual stresses caused by the friction stir welding of 5083 aluminum plates. Similar studies were developed by Staron et al. [31] and Preve'y et al. [32] on the friction stir welding of aluminum.

\subsection{Design of experiment (DOE)}

Due to that residual stresses produced by the FSW process plays an important role in designing the industrial equipment, optimization of the FSW process parameters which could 
influence on the residual stresses are very essential. Design of experiment (DOE) and statistical techniques are extensively used to optimize process parameters. Taguchi method is one of popular optimization techniques that could be used to optimize welding parameters.

Optimization of process parameters is a key step in the Taguchi technique to reach high quality without increasing the cost. This is because optimization of FSW process parameters can decrease the residual stress, which leads to improvement of performance characteristics. The optimum process parameters obtained from the Taguchi method are not sensitive to the variation of environmental conditions and other noise factors [33]. Generally, classical process parameter design is complex and not easy to solve. This is mainly true when the number of the process parameters increases, leading to a large number of experiments have to be carried out. To solve this problem, the Taguchi method with a special design of orthogonal arrays can be employed to study the entire process parameter space with a small number of experiments only [34]. The optimum combination of the process parameters can then be predicted [35].

\subsection{Goals and objectives of this study}

The main goal of this study is optimization of residual stresses produced by the FSW process on aluminum plates. By using the Taguchi method, the DOE is employed to optimize welding parameters including feed rate, rotational speed, pin diameter and shoulder diameter. The optimization process leads to residual stress minimization while the longitudinal residual stresses are measured by utilizing the $L_{C R}$ waves. The ultrasonic stress measurement results are also verified by employing the hole-drilling standard technique. By using statistical analysis of variance (ANOVA), it has been concluded that the most significant effect on the longitudinal residual stress peak is related to the feed rate while the pin and shoulder diameter have no dominant effect. In addition to the feed rate effect, the rotational speed variation also leads to changing the welding heat input which affect on the residual stress considerably.

\section{Theoretical Background}

\section{1. $L_{C R}$ method}

Different experimental setups can be used for residual stresses measurements fulfilled by the $L_{C R}$ waves. As a common configuration, three ultrasonic transducers with same frequency are 
used. The longitudinal waves are produced at the first critical angle by a transmitter (sender) transducer, then propagate parallel to the surface of tested material and finally are detected by two receiver transducers assembled in different distance from the sender. The reason of using two receiver transducers is decreasing the environmental effects, like ambient temperature, on the wave velocity. More experimental details of $L_{C R}$ waves can be found in several previous studies [18-24]. The relation between travel-time measured by the $L_{C R}$ wave and the corresponding uniaxial stress is derived by Egle and Bray [8] to be:

$$
\Delta \sigma=\frac{E}{L t_{0}}\left(t-t_{0}\right)
$$

In Eq. (1), $\Delta \sigma$ is stress change, $E$ is the elastic modulus and $L$ is the acoustoelastic coefficient (known as acoustoelastic constant) for longitudinal waves propagated in the direction of the applied stress field. The acoustoelastic constant would be measured by the uniaxial tensile test carried out over the samples extracted from the tested material. Also, $t$ is travel-time of the $L_{C R}$ wave, which is experimentally measured on the tested material while $t_{0}$ is travel-time for a homogeneous, isotropic, stress-free sample at the room temperature. Measuring the acoustoelastic constant along with the weld induced change in travel-time leads to determination of the stress variations produced by the welding process.

\subsection{Taguchi method}

Taguchi method is a powerful tool in quality optimization of different manufacturing processes. The traditional experimental designs involve in the average characteristics obtained from a numbers of experiments while the Taguchi method employs a special design of orthogonal array (OA) to examine the quality characteristics through a minimal number of experiments. The experimental results based on the OA are then transformed into the $\mathrm{S} / \mathrm{N}$ ratios to find the performance characteristics. Therefore, the Taguchi method concentrates on the effects of variations on quality characteristics, rather than on the averages. It means that, the Taguchi method makes the process performance insensitive to the variation of uncontrollable noise factors.

In this study, the Taguchi method is used to determine the optimal operational parameters of the FSW process to minimize welding residual stresses. The technique includes the following steps: (1) identifying the objectives of the experiment; (2) identifying the quality characteristic; 
(3) identifying the control factors that may affect on the quality characteristic; (4) selecting an appropriate $\mathrm{OA}$ and assign the factor levels; (5) performing experimental trials based on the configured OA; (6) determining the optimal levels of control factors based on the S/N ratios; (7) determining the significant factors that mostly affect the quality characteristic by the analysis of variance (ANOVA); (8) verifying the optimal operational parameters through validation experiments.

\section{Experimental Procedures}

\subsection{Sample Description}

The investigated process parameters of FSW includes feed rate, rotational speed, pin diameter and shoulder diameter. The Taguchi L8 orthogonal array is selected to evaluate the effects of process parameters on the welding residual stresses. Two levels are considered for each of the four process parameters, based on preliminary welding trials. Interacting influences between process parameters are not considered. Table 1 lists all the process parameter combinations investigated in this study.

All the samples S1-S8 (listed in Table 1) are manufactured by FSW process joining two $150 \times 100 \times 8 \mathrm{~mm}$ plates made from 5086 aluminum. The FSW tool is made from $\mathrm{H} 13$ steel which the design and dimensions are shown in the Fig. 1. The FSW is performed by using a vertical milling machine while the process parameters including feed rate, tool rotational speed, pin diameter and shoulder diameter are listed in Table 1 for each experimental sample. The tilt angle of FSW tool is equal to $2^{\circ}$ for all samples. During the FSW process, the workpiece is clamped at its sides by using a steel fixture and also supported at its bottom by a back plate.

\subsection{Measurement Devices}

Ultrasonic stress measurement using the $L_{C R}$ waves, which is considered in this study, has recently been confirmed in numerous studies [10-24]. A relatively correspondent experimental configuration is proposed in the previous works of Javadi et al [11,20-21] to produce the $L_{C R}$ waves usable for the ultrasonic stress measurement of plates. Using similar configuration, the ultrasonic measurement device (shown in Fig. 2) includes an ultrasonic box, laptop and time of flight (TOF) measuring equipment. Also, an automatic measuring table is needed to move TOF 
measuring equipment over the investigated plates with proper accuracy and stability. The automatic table is equipped with three step motors to be able to translate the ultrasonic transducers in 3 axes with resolution of $1 \mu \mathrm{m}$. The ultrasonic box is a portable ultrasonic testing device with sample rate of $100 \mathrm{MHz}$. The ultrasonic box is also equipped with an analogue to digital (A/D) converter, which is controlled by synchronization between the pulser signal and the internal clock. The internal clock works with the resolution of $1 \mathrm{~ns}$ which allows precise TOF measurement. The TOF measuring equipment includes three normal transducers assembled on an integrated wedge. The laser cutting (with consideration to the results presented by Eltawahni et al [36]) and CNC machining are employed to construct the wedge from poly methyl methacrylate (PMMA) material, under the trademark Plexiglas. The three-probe configuration is used, with one sender and two receivers in order to eliminate temperature effects on the travel time while frequency of the transducers is equal to $2 \mathrm{MHz}$. A constant and continues pressure, which is supplied by using a pneumatic cylinder, is needed to be exist over the wedge in order to keep constant thickness of ultrasonic couplant layer between the wedge and tested plate. There is also a couplant layer between the assembled transducers and their location in the wedge while the plastic screws support the constant pressure on the transducers.

\subsection{Evaluation of the acoustoelastic constant}

The standard uniaxial tensile test is employed to evaluate the acoustoelastic constant while a changed form of Eq. (1) could be used for the calculations:

$$
L=\frac{E}{\Delta \sigma \times t_{0}}\left(t-t_{0}\right)
$$

Tensile test specimens are extracted from the workpiece and would be exposed to the tensile test. During the tensile test, the TOF measuring equipments are assembled on the specimen in order to measure flight-time $(t)$ of the $L_{C R}$ wave. However, before starting the tensile test, the specimens should be stress relieved to determine the stress-free flight-time $\left(t_{0}\right)$ by TOF measurement. By using a tensile test standard machine, the tensile test $(\sigma)$ is increased step by step; meanwhile the flight-time $(t)$ is measured in each step. The elastic modulus $(E)$ could also be measured by using the tensile test results or obtaining from the material tables. As a result, the acoustoelastic constant $(L)$ would be calculated. 
In this study, the tensile test specimens are extracted from the weld zone and parent material (PM) to measure the $E$ and $L$ in each of these zones. The tensile test specimens are produced according to the Sheet type (0.5 in. wide) sample presented in the ASTM: E8 standard.

As a result, the acoustoelastic constant is measured equal to 3.31 and 4.09 in the PM and weld zone respectively. The measurement results are comparable with those reported by Sadeghi et al [12] in the acoustoelastic constant measurement of aluminum plates.

\subsection{Hole-drilling measurement to validate ultrasonic stress evaluation}

The capability of using the $L_{C R}$ waves in the ultrasonic stress measurement has been confirmed in many previous studies [11-24]. However, the $L_{C R}$ method is still considered as an underdeveloped technique, which needs to be verified by employing another stress measurement method. In this study, the ultrasonic stress measurement results are validated by utilizing the hole-drilling measurement. The hole-drilling method is carried out in three different points (as shown in Fig. 3) according to the characterizations described in ASTM: E837. This semidestructive technique measures the strains relaxed by incremental drilling of a small hole [37]. The strains are measured using a strain gauge rosette (Fig. 3) after each depth increment and the stress field is then calculated employing equations established by ASTM: E837.

\section{Results and Discussion}

\subsection{Ultrasonic stress measurement}

The following steps are accomplished to measure longitudinal residual stresses in samples S1S8 (described in Table 1) by using the $L_{C R}$ ultrasonic waves:

1- The measurement devices (section 3.2) are employed to measure TOF in sample S1 while the $L_{C R}$ wave is propagated parallel to the FSW direction, which leads to longitudinal stress measurement.

2- The tensile test specimens are prepared from the weld and parent material (PM) zones extracted from the sample S1.

3- The value $t_{0}$ is evaluated directly on the stress-free samples, which are the tensile test specimens prepared in step 2. The stress-free situation is created by employing a stress relieving heat treatment. 
4- The elastic modulus $(E)$ is measured by using tensile test on the specimens prepared in step 2 .

5- The acoustoelastic constant is measured by using a uniaxial tensile test and Eq. (2) in the weld and PM zones separately (section 3.3).

6- By putting the results of step 1-5 in Eq. (1), longitudinal residual stresses of sample S1 could be calculated.

7- Comparing the ultrasonic stress measurement results with those obtained from the holedrilling measurement (shown in Fig 4) could be considered as the verification of ultrasonic measurement while a good agreement has been observed between the results.

8- The verified ultrasonic method could be used for stress measurement of the other samples (S2-S8); hence steps 1 to 6 would be applied on samples S2-S8 to evaluate longitudinal residual stresses of them (shown in Fig 5).

It can be observed that the peak of longitudinal residual stress for all samples S1-S8 is occurred in the advancing side (AS) of FSW (also reported by Sadeghi et al. [12]). The higher residual stresses in AS, in comparison with the retreating side (RS), is because of non-symmetric temperature distribution of FSW [38]. Furthermore, the major part of material flow is produced in the AS, hence stirred zone (SZ) stretches into the AS leading to higher residual stress [39].

\subsection{Process parameter optimization}

The aim of this investigation is to evaluate the effects of FSW process parameters on the welding residual stresses. Towards this end, the analysis of variance (ANOVA) is performed using the longitudinal residual stress as the response parameter following standard ANOVA procedure [35]. Table 2 summarizes the ultrasonic stress measurement results related to the influence of FSW process parameters on the residual stress peak in samples S1-S8. It can be observed that the residual stress peak of S3 is the maximum among the other experimental samples.

To determine which FSW process parameters have the maximum effect on the residual stress, it is needed to find signal-to-noise $(\mathrm{S} / \mathrm{N})$ ratio. There are three categories of performance characteristics employed for analyzing S/N ratio; the lower the better, the higher the better and the nominal the better. The smaller-the-better characteristic of the residual stress is considered in this study. Thus, the $\mathrm{S} / \mathrm{N}$ ratio $\eta_{i j}$ for $\mathrm{i}$ th experiment in the $\mathrm{j}$ th test is given by: 


$$
\eta_{i j}=-10 \log \left(\frac{1}{n} \sum_{j=1}^{n} \frac{1}{y_{i j}^{2}}\right)
$$

In Eq. (3); $y_{i j}$ is ith experiment at the $\mathrm{j}$ th test and $n$ is the number of tests.

The main effects plots show how each factor affects the response characteristic, which is the longitudinal residual stress. A main effect is present when different levels of a factor affect the characteristic differently. In this study, MINITAB 16 is used to create the main effects plot by plotting the characteristic average for each factor level (Fig. 6). These averages are the same as those displayed in the response table (Table 3). A line connects the points for each factor. Both figure and table show that the feed rate has the maximum effect on the residual stress.

The purpose of ANOVA is to investigate which FSW process parameters notably affect the quality characteristic. This is fulfilled by separating the total variability of the $\mathrm{S} / \mathrm{N}$ ratios, which is evaluated by the sum of the squared deviations from the total mean of the $\mathrm{S} / \mathrm{N}$ ratio, into contributions by each FSW process parameter and the error [40-41]. The results of ANOVA for FSW process optimization are shown in Table 4. In the Taguchi analysis, the p-values (P) are used to determine which of the experimental parameters has significant effect. The p-value (P) for each factor is calculated by the software and compared with the level of significance, $\alpha$-level. If the p-value for the squared effects is less than $\alpha$-level (which is usually considered to be 0.05 ) then there is a significant quadratic effect. From Table 4, it can be concluded that the feed rate and rotational speed have significant effect on the residual stress because the p-value (P) is less than 0.05 . However, the feed rate with $\mathrm{P}$ equal to 0.002 has the highest effect on the residual stress. Furthermore, the pin and shoulder diameter have no significant effect on the residual stress because the p-value (P) is considerably higher than the $\alpha$-level.

Since the variations of feed rate and rotational speed lead to changing the welding heat input considerably, dominate effect of these parameters on the residual stresses was expectable (similar results were also reported by Lombard et al [42]). However, employing the Taguchi method to investigate this effect was not considered in the previous studies. Furthermore, the combination of using Taguchi and $L_{C R}$ ultrasonic method $\left(T L_{C R}\right)$, which is considered as the main contribution of this study, leads to a comprehensive investigation on the FSW residual stresses. Regarding to the high amount of measurement points in the ultrasonic stress measurement, the reliability of Taguchi optimization is considerably increased by employing the $T L_{C R}$ method in the residual stress evaluation of FSW process. 


\section{Conclusions}

The main goal of this study is process optimization of FSW to determine which process parameter has the highest effect on the longitudinal residual stresses in aluminum plates. Taguchi method, the ultrasonic stress measurement by employing the $L_{C R}$ waves and the hole-drilling method are employed to reach this goal. According to the achieved results, it can be concluded that:

1) The peak of longitudinal residual stress is occurred in the advancing side (AS) of FSW. The position of peak could be accurately determined by employing the $L_{C R}$ ultrasonic method.

2) The most significant effect on the longitudinal residual stress peak is related to the feed rate (in comparison with the other process parameters of FSW).

3) After the feed rate, the rotational speed has considerable effect on the residual stress peak.

4) The pin and shoulder diameter of FSW tool has no dominant effect on the longitudinal residual stress peak.

The $T L_{C R}$ method, which is combination of using Taguchi and the $L_{C R}$ ultrasonic method, showed a good reliability in the residual stress evaluation of aluminum plates joined by the FSW process.

\section{References}

[1] Rossini NS, Dassisti M, Benyounis KY, Olabi AG. Methods of measuring residual stresses in components. J Materials \& Design 2012; 35:572-88.

[2] Olabi AG, Hashmi MSJ. The effect of post-weld heat-treatment on mechanical-properties and residual-stresses mapping in welded structural steel. Journal of Materials Processing Technology 1995; 55: 117-22.

[3] Olabi AG, Hashmi MSJ. The Microstructure and Mechanical Properties of Low Carbon Steel Welded Components After the Application of PWHTs. Journal of Materials Processing Technology 1996; 56:88-97.

[4] Olabi AG, Hashmi MSJ. Stress Relief Procedures for Low Carbon Steel (1020) Welded Components. Journal of Materials Processing Technology 1996; 56:552-62.

[5] Olabi AG, Hashmi MSJ. Effects of the stress-relief conditions on a martensite stainlesssteel welded component. Journal of Materials Processing Technology 1998; 77: 216-25. 
[6] Olabi AG, Casalino G, Benyounis KY, Rotondo A. Minimisation of the residual stress in the heat affected zone by means of numerical methods. Materials and Design 2007; 28: 2295-302.

[7] Crecraft DI. The Measurement of Applied and Residual Stresses in Metals Using Ultrasonic Waves. J Sound Vib 1967. 5: 173-92.

[8] Egle DM, Bray DE. Measurement of Acoustoelastic and Third-Order Elastic Constants for Rail Steel. J Acoust Soc Am 1976. 60: 741-4.

[9] Tang W, Bray DE. Stress and Yielding Studies Using Critically Refracted Longitudinal Waves. NDE Engineering Codes and Standards and Material Characterization, Montreal: ASME Pressure Vessels Piping Conference, 1996; 322: 41-8.

[10] Bray DE, Tang W. Subsurface stress evaluation in steel plates and bars using the LCR ultrasonic wave. Nuclear Engineering and Design 2001; 207: 231-40.

[11] Javadi Y, Akhlaghi M, Najafabadi MA. Using Finite Element and Ultrasonic Method to Evaluate Welding Longitudinal Residual Stress through the Thickness in Austenitic Stainless Steel Plates. J Materials and Design 2013; 45: 628-42.

[12] Sadeghi S, Najafabadi MA, Javadi Y, Mohammadisefat M. Using ultrasonic waves and finite element method to evaluate through-thickness residual stresses distribution in the friction stir welding of aluminum plates. Materials and Design 2013; 52:870-880.

[13] Javadi Y, Pirzaman HS, Raeisi MH, Najafabadi MA. Ultrasonic inspection of a welded stainless steel pipe to evaluate residual stresses through thickness. Materials and Design 2013; 49: 591-601.

[14] Belahcene F, Lu J. Determination of residual stress using critically refracted longitudinal waves and immersion mode. The Journal of Strain Analysis for Engineering Design 2002; 37: 13-9.

[15] Palanichamy P, Vasudevan M, Jayakumar T. Measurement of residual stresses in austenitic stainless steel weld joints using ultrasonic technique. Science and Technology of Welding and Joining 2009; 14: 166-71.

[16] Javadi Y, Ahmadi Najafabadi M. Comparison between contact and immersion ultrasonic method to evaluate welding residual stresses of dissimilar joints. Materials and Design 2013; 47: 473-82.

[17] Javadi Y, Najafabadi MA, Akhlaghi M. Comparison between Contact and Immersion Method in Ultrasonic Stress Measurement of Welded Stainless Steel Plates. Journal of Testing and Evaluation 2013; 41: 1-10.

[18] Javadi Y, Pirzaman HS, Raeisi MH, Najafabadi MA. Ultrasonic Evaluation of Welding Residual Stresses in Stainless Steel Pressure Vessel. Journal of Pressure Vessel Technology 2013; 135: 041502: 1-6.

[19] Javadi Y, Najafabadi MA, Akhlaghi M. Residual Stress Evaluation in Dissimilar Welded Joints Using Finite Element Simulation and the LCR Ultrasonic Wave. Russian Journal of Nondestructive Testing 2012; 48: 541-52. 
[20] Javadi Y, Afzali O, Raeisi MH, Najafabadi MA. Nondestructive Evaluation of Welding Residual Stresses in Dissimilar Welded Pipes. Journal of Nondestructive Evaluation 2013; 32: $177-87$.

[21] Javadi Y, Akhlaghi M, Najafabadi MA. Nondestructive Evaluation of Welding Residual Stresses in Austenitic Stainless Steel Plates. Research in Nondestructive Evaluation 2013. DOI: $10.1080 / 09349847.2013 .822134$.

[22] Sattari-Far I, Javadi Y. Influence of welding sequence on welding distortions in pipes. International Journal of Pressure Vessels and Piping 2008; 85: 265-274.

[23] Javadi Y. Ultrasonic Measurement of Hoop Residual Stress in Stainless Steel Pipes. Journal of Manufacturing and Industrial Engineering 2013; 1-2: 1-6.

[24] Javadi Y, Hloch S. Employing the LCR Waves to Measure Longitudinal Residual Stresses in Different Depths of a Stainless Steel Welded Plate. Advances in Materials Science and Engineering 2013; 2013: 746187.

[25] Thomas WM, Nicholas ED, Needham JC, Murch MG, Templesmith P, Dawes CJ. Friction Stir Welding. International Patent Application No. PCT/GB92102203 and Great Britain Patent Application No. 9125978.8, 1991.

[26] Rhodes CG, Mahoney MW, Bingel WH, Spurling RA, Bampton CC. Effects of friction stir welding on microstructure of 7075 aluminum. Scripta Materialia 1997; 31: 69-75.

[27] Liu HJ, Fujii H, Maeda M, Nogi K. Tensile properties and fracture locations of frictionstir-welded joints of 2017-T351 aluminium alloy. Journal of Materials Processing Technology 2003; 142: 692-6.

[28] Guerra M, Schmidt C, McClure JC, Murr LE, Nunes AC. Flow patterns during friction stir welding. Materials Characterization 2002; 49: 95-101.

[29] Bussu G, Irving PE. The role of residual stress and heat affected zone properties on fatigue crack propagation in friction stir welded 2024-T351 aluminium joints. Int J Fatigue 2003; 25: 77-88.

[30] Peel M, Steuwer A, Preuss M, Withers PJ. Microstructure, Mechanical properties and residual stresses as a function of welding speed in alluminium AA5083 friction stir welds. Acta Mater 2003; 51: 4791-801.

[31] Staron P, Kocak M, Williams S. Residual stresses in friction stir welded Al sheets. Appl Phys A: Mater Sci Process 2002; 74: 1161-2.

[32] Preve'y P, Mahoney M. Improved fatigue perform of friction stir welds with low plasticity burnishing: residual stress design and fatigue perform assessment. Mater Sci Forum 2003; 426: 2933-40.

[33] Tarng YS, Juang SC, Chang CH. The use of grey-based Taguchi methods to determine submerged arc welding process parameters in hard facing. J Mater Process Technol 2002; 128(1-3):1-6.

[34] Montgomery DC. Design and analysis of experiments. Singapore: Wiley; 1991.

[35] Ross PJ. Taguchi techniques for quality engineering. New York: McGraw-Hill Book Company; 1988. 
[36] Eltawahni HA, Olabi AG, Benyounis KY. Effect of process parameters and optimization of $\mathrm{CO} 2$ laser cutting of ultra high-performance polyethylene. Materials and Design 2010; 31: 4029-38.

[37] Anawa EM, Olabi AG. The application of the hole drilling method to define the residual stress of dissimilar laser welded components. J Appl Mech Mater 2007; 7-8: 133-8.

[38] Buffa G, Ducato A, Fratini L. Numerical procedure for residual stresses prediction in friction stir welding. Finite Elements in Analysis and Design 2011; 47: 470-6.

[39] Asadi P, Mahdavinejad RA, Tutunchilar S. Simulation and experimental investigation of FSP of AZ91 magnesium alloy. Materials Science and Engineering A 2011; 528: 646977.

[40] Anawa EM, Olabi AG. Effect of laser welding conditions on toughness of dissimilar welded components. J Appl Mech Mater 2006; 5-6: 375-80.

[41] Anawa EM, Olabi AG. Using Taguchi method to optimize welding pool of dissimilar laser-welded components. J Opt Laser Technol 2008; 40: 379-88.

[42] Lombard H, Hattingh DG, Steuwer A, James MN. Effect of process parameters on the residual stresses in AA5083-H321 friction stir welds. Materials Science and Engineering A 2009; 501: 119-124. 


\section{List of table captions}

Table 1. Investigated experimental samples based on the Taguchi L8 orthogonal array

Table 2. Influence of FSW process parameters on the residual stress peak

Table 3. Response table for signal-to-noise ratios (the smaller is better)

Table 4. The analysis of variance for FSW optimization 


\section{List of figure captions}

Fig.1. Design and dimensions of the FSW tool

Fig. 2. The $L_{C R}$ measurement devices

Fig. 3. Hole-drilling setup on aluminum plates

Fig. 4. Verification of the ultrasonic stress measurement by employing the hole-drilling method on sample $\mathrm{S} 1$

Fig. 5. Longitudinal residual stress measured by the ultrasonic method on samples 1-8

Fig. 6. The S/N ratios for the FSW process parameters 